\title{
Suplementação de vitamina A em dietas para Kinguios (Carassius auratus)
}

\section{Vitamin A supplementation in diets for Goldfish (Carassius auratus)}

\author{
Edionei Maico Fries $^{1 *}$; Alis Correia Bittarello²; Deividy Miranda da Silva ${ }^{3}$; \\ Altevir Signor ${ }^{4}$; Wilson Rogério Boscolo ${ }^{4}$; Aldi Feiden ${ }^{4}$
}

\begin{abstract}
Resumo
A vitamina A é importante para o correto desenvolvimento e funcionamento do organismo, figurando em diversas funções metabólicas. Foi conduzido um experimento com o objetivo de avaliar a suplementação de vitamina A em dietas para kinguio Carassius auratus. O delineamento experimental foi inteiramente casualizado com seis tratamentos e quatro repetições $(0,2.000,4.000,8.000,16.000$ e $32.000 \mathrm{UI}$ de vitamina $\mathrm{A} \mathrm{kg}^{-1}$ de dieta) em 24 tanques rede experimentais, com capacidade para 150 litros de volume útil, confeccionados em malha sombrite, instalados no interior de um tanque circular de alvenaria com capacidade para $25 \mathrm{~m}^{3}$ de água. Foram utilizados 192 peixes com peso inicial médio de $6,66 \pm 0,57 \mathrm{~g}$ e comprimento inicial médio de $7,30 \pm 0,33 \mathrm{~cm}$, alimentados às $8 \mathrm{~h}, 11 \mathrm{~h}, 14 \mathrm{~h}$ e $17 \mathrm{~h}$ ate a saciedade. Foram avaliados os efeitos sobre o desempenho produtivo e intensidade de coloração vermelho-amarelada da pele dos peixes. A análise de Linear Response Plateau do ganho de peso, comprimento padrão e total, conversão alimentar aparente e taxa de crescimento diário versus níveis de vitamina A demonstrou estimativa de exigência de $2.624,2.385$ e $2.358,4.381$ e 2.866 UI de vitamina A por kg de ração, respectivamente. Os dados indicam que aparentemente esta vitamina tem pouca ou nenhuma influência sobre a intensidade da cor da pele do kinguio.
\end{abstract}

Palavras-chave: Acetato de retinol, coloração, nutrição, peixes ornamentais

\begin{abstract}
Vitamin A is important to the proper development and functioning of the body, appearing in several metabolic functions. This experiment was conducted with aim evaluate to the vitamin A supplementation in diets for Goldfish Carassius auratus. The experimental was completely randomized with six treatments $\left(0,2.000,4.000,8.000,16.000\right.$ and $32.000 \mathrm{IU}$ of vitamin $\mathrm{A} \mathrm{kg}^{-1}$ diet $)$ with four repetitions into 24 experimental cages, with capacity for 150 liters useful volume, made of mesh shading, installed inside a masonry circular tank with a capacity of $25 \mathrm{~m}^{3}$ of water. 192 fish with an average initial weight of $6.66 \pm 0.57 \mathrm{~g}$ and initial length of $7.30 \pm 0.33 \mathrm{~cm}$, feed at $8 \mathrm{~h}, 11 \mathrm{~h}, 14 \mathrm{~h}$ and $17 \mathrm{~h}$ at satiety were used. Evaluated the effects on productive performance and intensity of yellow-red coloring of the skin of the fish were. The Linear Response Plateau analysis of weight gain and length total, feed conversion and daily growth rate versus vitamin A requirement estimate showed 2,624, 2,385 and 2,358, 4,381 and 2,866 IU of vitamin A per kg diet, respectively. The data indicated that this vitamin has little or no influence on the intensity of the skin color of the Goldfish.
\end{abstract}

Key words: Retinol acetate, coloring, nutrition, ornamental fish

${ }^{1}$ Eng $^{0}$ de Pesca, Discente do Programa de Pós-Graduação em Recursos Pesqueiros e Engenharia de Pesca, Toledo, PR. E-mail: edioneifries@hotmail.com

${ }^{2}$ Zootecnista, M.e em Recursos Pesqueiros e Engenharia de Pesca, Discente do Programa de Pós-Graduação em Zootecnia, UNESP, Botucatu, SP. E-mail: alis@zootecnista.com.br

${ }^{3}$ Discente do Curso de Graduação em Engenharia de Pesca, UNIOESTE, Toledo, PR. E-mail: deividy.miranda@hotmail.com

${ }^{4}$ Profs., Curso de Engenharia de Pesca, UNIOESTE, Toledo, PR. E-mail: aldifeiden@gmail.com; altevir.signor@gmail.com; wilsonboscolo@hotmail.com

* Autor para correspondência 


\section{Introdução}

A piscicultura ornamental é uma atividade bastante lucrativa que no Brasil se destaca em crescimento e exportações (SILVA; SCHULZ, 2006). É um mercado dinâmico e ávido por novidades, entre as quais podem ser destacadas as novas espécies e linhagens de peixes utilizadas para ornamentação, em função de sua beleza e raridade, bem como dietas mais eficientes para intensificar a pigmentação da pele e a saúde dos peixes.

Dentre as espécies com interesse para ornamentação está o C. auratus, conhecido popularmente por kinguio, estando entre os peixes mais cultivados, ocupando lugar de destaque na comercialização mundial de peixes ornamentais, devido a sua beleza, rusticidade, adaptação ao manejo e as condições ambientais, além da alta prolificidade (SILVA; SCHULZ, 2006).

Vários estudos estão sendo desenvolvidos com o objetivo de maximizar a lucratividade no cultivo de peixes ornamentais, com o intuito de reduzir mortalidade, melhorar a conversão alimentar e aumentar o desempenho no cultivo intensivo. Porém, ainda hoje, pode ser visto o uso generalizado de rações de peixes de corte na produção de peixes ornamentais, que não suprem adequadamente as exigências nutricionais dessas espécies. É necessário o desenvolvimento de pacotes tecnológicos, com o estabelecimento de índices zootécnicos e o desenvolvimento de dietas que atendam às exigências nutricionais para as diversas espécies cultivadas para a aquariofilia (ZUANON; SALARO; FURUYA, 2011).

Anutrição vitamínica ainda não é completamente ajustada nem mesmo para as diversas espécies de peixes para corte, apesar de essencial para saúde, crescimento e reprodução de qualquer organismo aquático. Dentre as vitaminas que precisam ser incluídas nas dietas destaca-se a vitamina A, que é integrante do grupo das vitaminas lipossolúveis, juntamente com as vitaminas D, E e K. Ela Exerce papel fundamental no estímulo para o crescimento de novas células, fator essencial para o crescimento adequado e na manutenção do tecido epitelial (produção de muco por este tecido) (HALVER, 2002). A vitamina A também tem grande influência na resistência a infecções, na reprodução e é vital para uma visão normal (HALVER, 2002).

A vitamina $\mathrm{A}$, assim como as demais vitaminas lipossolúveis, merece atenção em relação à suplementação adequada, pois a ingestão acima das necessidades metabólicas pode provocar sintomas de hipervitaminose, já que seu excedente não é prontamente excretado pelo organismo e fica armazenado no fígado dos animais (MCDOWELL, 1989; TACON, 1991; HALVER, 2002).

Apesar de sua reconhecida importância, a vitamina A tem sua exigência determinada e quantificada para poucas espécies de peixes, entre elas: Oncorhynchus mykiss (KITAMURA et al., 1967), Ictalurus punctatus (DUPREE, 1970), Cyprinus carpio (AOE et al., 1968), Seriola lalandi (SHIMENO, 1991), Epinephelus tauvina (SHAIK MOHAMED et al., 2003), Hippoglossus hippoglossus (MOREN et al., 2004), Morone chrysops x Morone saxatilis (HEMRE et al., 2004), Paralichthys olivaceus (HERNANDEZ et al., 2005), Oreochromis niloticus x Oreochromis aureus (HU et al., 2006), constantes no NRC (2011).

Considerando, então, a importância da vitamina A nas atividades vitais do organismo e seu potencial tóxico quando em quantidades acima das exigências, o objetivo deste estudo foi avaliar a suplementação de vitamina A em dietas para kinguio C. auratus.

\section{Material e Métodos}

O experimento foi realizado na Universidade Estadual do Oeste do Paraná, Campus Toledo/PR. Foram utilizados 192 juvenis de $C$. auratus, com peso e comprimento inicial médio de $6,66 \pm 0,57 \mathrm{~g}$

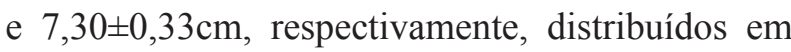
24 tanques-rede experimentais confeccionados em malha sombrite instalados no interior de um tanque 
circular de alvenaria com capacidade para $25 \mathrm{~m}^{3}$ de volume útil, com aeração constante, em um delineamento inteiramente casualizado, com seis tratamentos e quatro repetições. Foi considerada uma unidade experimental um tanque-rede com oito peixes.

As rações foram formuladas com base nas exigências de peixes onívoros propostos pelo NRC (2011). A suplementação da vitamina A nas rações experimentais ocorreu através da elaboração de seis suplementos mineral e vitamínico apresentando $0,2.000,4.000,8.000,16.000$ e 32.000 UI de vitamina $\mathrm{A} \mathrm{kg}^{-1}$ de dieta (Tabela 1), suplementada pelo acetato de retinol (Microvit ${ }^{\mathrm{TM}}$ A Supra 1000) com concentração de 1.000.000 UI g ${ }^{-1}$ de vitamina A. Este suplemento foi adicionado à uma dieta basal contendo $36 \%$ de proteína bruta e $3.500 \mathrm{kcal} \mathrm{kg}^{-1} \mathrm{de}$ energia digestível (Tabela 2).

Tabela 1. Formulação do suplemento mineral e vitamínico incorporado nas dietas experimentais.

\begin{tabular}{lclllll}
\hline & \multicolumn{7}{c}{ Tratamentos $\left(\mathrm{m} \mathrm{kg}^{-1}\right.$ VIT A) } \\
\cline { 2 - 7 } \multicolumn{1}{c}{ Nutrientes } & 0 & 2000 & 4000 & 8000 & 16000 & 32000 \\
\hline Vitamina A & 0,000 & 0,012 & 0,024 & 0,048 & 0,096 & 0,192 \\
Vitamina D3 & 0,036 & 0,036 & 0,036 & 0,036 & 0,036 & 0,036 \\
Vitamina E & 1,800 & 1,800 & 1,800 & 1,800 & 1,800 & 1,800 \\
Vitamina K3 MNB & 0,206 & 0,206 & 0,206 & 0,206 & 0,206 & 0,206 \\
Vitamina B1 & 0,122 & 0,122 & 0,122 & 0,122 & 0,122 & 0,122 \\
Vitamina B2 & 0,150 & 0,150 & 0,150 & 0,150 & 0,150 & 0,150 \\
Vitamina B6 & 0,110 & 0,110 & 0,110 & 0,110 & 0,110 & 0,110 \\
Vitamina B12 & 0,024 & 0,024 & 0,024 & 0,024 & 0,024 & 0,024 \\
Vitamina C & 5,143 & 5,143 & 5,143 & 5,143 & 5,143 & 5,143 \\
Niacina & 0,612 & 0,612 & 0,612 & 0,612 & 0,612 & 0,612 \\
Pantotenato de Cálcio & 0,306 & 0,306 & 0,306 & 0,306 & 0,306 & 0,306 \\
Biotina & 0,300 & 0,300 & 0,300 & 0,300 & 0,300 & 0,300 \\
Ácido Fólico & 0,037 & 0,037 & 0,037 & 0,037 & 0,037 & 0,037 \\
Inositol & 0,918 & 0,918 & 0,918 & 0,918 & 0,918 & 0,918 \\
Cloreto de Colina & 5,000 & 5,000 & 5,000 & 5,000 & 5,000 & 5,000 \\
Sulfato de Cobre pentahidratado & 0,432 & 0,432 & 0,432 & 0,432 & 0,432 & 0,432 \\
Sulfato de Ferro monohidratado & 1,600 & 1,600 & 1,600 & 1,600 & 1,600 & 1,600 \\
Sulfato de Manganês & 1,154 & 1,154 & 1,154 & 1,154 & 1,154 & 1,154 \\
Sulfato de Zinco & 2,057 & 2,057 & 2,057 & 2,057 & 2,057 & 2,057 \\
Iodato de cálcio & 0,008 & 0,008 & 0,008 & 0,008 & 0,008 & 0,008 \\
Selenito de sódio & 0,007 & 0,007 & 0,007 & 0,007 & 0,007 & 0,007 \\
Sulfato de Cobalto & 0,017 & 0,017 & 0,017 & 0,017 & 0,017 & 0,017 \\
\hline Subtotal & 20,039 & 20,051 & 20,063 & 20,087 & 20,135 & 20,231 \\
\hline Propionato & 0,600 & 0,600 & 0,600 & 0,600 & 0,600 & 0,600 \\
Veículo & 9,361 & 9,349 & 9,337 & 9,313 & 9,265 & 9,169 \\
\hline Total & 30,000 & 30,000 & 30,000 & 30,000 & 30,000 & 30,000 \\
\hline
\end{tabular}

Fonte: Elaboração dos autores.

Para a elaboração das rações, os alimentos foram moídos individualmente em moinho tipo martelo (HAYASHI et al., 1999), com peneira de malha 0,5 $\mathrm{mm}$. Em seguida, os ingredientes foram misturados e homogeneizados manualmente, posteriormente foi acrescido o suplemento mineral e vitamínico, diluindo-se a respectiva quantidade deste em uma pequena fração da mistura e, posteriormente, homogeneizados com o restante da ração e umedecida com $22 \%$ de água para realização do 
processo de extrusão com matriz de $1,2 \mathrm{~mm}$, secas por 24 horas em estufa de ventilação forçada a 55 ${ }^{\circ} \mathrm{C}$. O arraçoamento foi realizado quatro vezes ao dia às $8,11,14$ e 17h, ad libitum.

Os peixes foram mantidos em ambiente com água proveniente de poço artesiano e durante o período experimental apenas a água perdida por evaporação foi reposta. O oxigênio dissolvido médio de $5,65 \pm 0,60 \mathrm{mg} \mathrm{L}^{-1}$, temperatura de $19,95 \pm 2,73^{\circ} \mathrm{C}$, condutividade elétrica de 95,65 $\pm 8,50 \mu \mathrm{S} \mathrm{cm}^{-1}$ e

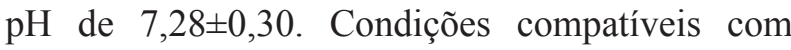
a faixa de conforto para a espécie. Os kinguios são relativamente tolerantes as variações dos parâmetros físicos e químicos da água (SILVA; SCHULZ, 2006), tendo preferência pelo pH neutro ou ligeiramente alcalino e temperatura variando entre 15 e $24^{\circ} \mathrm{C}$ (SCHÜMER, 2002).

Tabela 2. Composição percentual e nutricional utilizados na elaboração da dieta experimental.

\begin{tabular}{llll}
\hline Ingredientes & $\%$ & Nutrientes & $\%$ \\
\hline Soja farelo 48\% & 47,071 & Amido & 19,274 \\
Vísceras farinha aves & 20,000 & Arginina total & 2,578 \\
Milho grão & 16,711 & Cálcio & 1,048 \\
Arroz quirera & 10,017 & Energia digestível & 3500 \\
Óleo de soja & 5,381 & Fibra bruta & 2,778 \\
Suplemento (min. + vit.) & 0,500 & Fósforo total & 0,886 \\
Antioxidante (BHT) & 0,020 & Gordura & 9,000 \\
Sal & 0,300 & Lisina total & 2,096 \\
& & Met+ cistina total & 1,150 \\
& & Metionina total & 0,589 \\
& & Proteína bruta & 36,000 \\
& & Treonina total & 1,465 \\
\end{tabular}

Fonte: Elaboração dos autores.

Ao final do período experimental de 88 dias, os peixes permaneceram por 24 horas em jejum para o esvaziamento do trato gastrointestinal, posteriormente, foram insensibilizados em solução de benzocaína $(87,5 \mathrm{mg} / \mathrm{L})$ (BITTENCOURT et al., 2012), sendo contados, pesados e medidos para determinação dos parâmetros zootécnicos. Foram avaliados: peso final, comprimento final, ganho de peso, conversão alimentar aparente [alimento consumido (g)/ganho em peso $(\mathrm{g})$ ], sobrevivência e taxa de crescimento diário [LN(peso final)-LN(peso inicial)*100/tempo experimento].

A fim de avaliar a intensidade da coloração vermelho-amarelado resultante da ingestão de dietas suplementadas com diferentes níveis de vitamina A, os peixes foram fotografados no final do período de experimentação, de acordo com metodologia preconizada por Rezende et al. (2012), a qual está descrita a seguir.

Para que a variação diurna da incidência luminosa indireta do sol, não interferisse nas cores, as fotografias digitais foram tiradas em sala fechada, utilizando apenas iluminação artificial, através de uma lâmpada incandescente de 100 watts e outra fluorescente de 60 watts, posicionadas $80 \mathrm{~cm}$ acima da superfície de acomodação dos peixes.

Os registros foram realizados com câmera digital (SAMSUNG SL202 de 10.2 Megapixels, sensor de imagem $1 \backslash 2$ 33" CCD), e analisados pelo programa Adobe Photoshop ${ }^{\circledR} \mathrm{CS} 4$, com a modificação apresentada a seguir, a câmera foi posicionada a $35 \mathrm{~cm}$ do peixe a ser fotografado. A coloração da 
pele dos peixes foi quantificada utilizando o sistema de coordenadas Hunter e CMYK (\%). No sistema de cores CMYK, as cores são decompostas em percentuais das cores basais, ciano (C), magenta (M), amarelo (Y) e preto (K). Pelo fato de o sistema de cores CMYK apresentar variáveis não paramétricas, Vieira e Hoffmann (1989) sugerem a transformação de variáveis não paramétricas em variáveis paramétricas, através da equação: $\mathrm{C}_{(\mathrm{rad})}=$ $\operatorname{arcsen} .\left[\mathrm{C}_{(\%)} / 100\right]^{0,5}$. Onde, $\mathrm{C}_{(\%)}=$ representa valor percentual da cor observada.

Inicialmente as fotografias passaram por equilíbrio de cores, através do software Adobe Photoshop ${ }^{\circledR}$ versão CS4 (em inglês), utilizando os comandos no menu [Image]-[Adjustments]-[auto levels]. Para a obtenção dos valores percentuais referentes às cores amarela e magenta só poderão ser obtidos no modo de exibição de cores CMYK, o qual pode ser selecionado no menu [Image]-[Mode][CMYK color]. Em seguida, usando o comando no menu [File]-[Save As], as fotos equilibradas foram salvas identificando-se no nome do arquivo, a qual tratamento e repetição se refere o peixe fotografado.

A partir dos dados do sistema de coordenadas Hunter, foram calculados os valores referentes à croma $\left(\mathrm{C}^{*}{ }_{\mathrm{ab}}\right)$ que expressa à intensidade e claridade de uma cor, e é dada pela equação: $\mathrm{C}_{\mathrm{ab}}^{*}=\left(\mathrm{a}^{* 2}+\right.$ $\left.\mathrm{b}^{* 2}\right)^{1 / 2}$ e matiz $\left(\mathrm{H}^{*}{ }_{\mathrm{ab}}\right)$, a tonalidade expressa à relação entre o vermelho e o amarelo que é calculada pela equação: $\mathrm{H}_{\mathrm{ab}}=\tan ^{-1}\left(\mathrm{~b}^{*} / \mathrm{a}^{*}\right)$ (NICKELL; BROMAGE, 1998). A tonalidade é uma medida angular onde $0^{\circ}$ indica uma tonalidade vermelha e $90^{\circ}$ uma tonalidade amarela.

As variáveis encontradas foram submetidas à análise de variância unifatorial a um nível de significância de 5\%, com a checagem dos pressupostos de normalidade dos resíduos pelo teste de Shapiro-Wilk e homocedasticidade de variância pelo teste de Levene's. Para identificar as fontes de variação detectadas foi utilizado o teste de comparação de médias de Duncan e em caso de diferenças foi utilizada a análise de regressão pelo programa estatístico SAEG (UFV, 1997).

\section{Resultados e Discussão}

A suplementação de vitamina A na dieta de kinguios exerceu influência sobre o ganho de peso, crescimento e consumo de ração destes peixes, expresso nas variáveis de ganho de peso médio, comprimento padrão e total, conversão alimentar aparente e taxa de crescimento diário, obtidos no final do período experimental (Tabela 3). Com o incremento nos níveis de vitamina A nas rações, foi observado efeito quadrático $(\mathrm{P}<0,05)$ sobre o ganho de peso médio, comprimento padrão e total médio, conversão alimentar aparente e taxa de crescimento diário.

Os peixes que receberam a dieta suplementada com 4.000 UI de vitamina A por kg, tiveram o maior ganho de peso médio. No entanto, pelo modelo descontínuo LRP (Linear Response Plateau), estima como nível ideal de suplementação de 2.624 UI de vitamina A por $\mathrm{kg}$, nesse ponto ocorre um platô (Figura 1), correspondendo a um ganho de peso de 4,89 gramas.

O NRC (2011), baseado no estudo de Aoe et al. (1968) recomenda de 4.000 a $20.000 \mathrm{UI} \mathrm{kg}^{-1}$ de vitamina A para a carpa comum (Cyprinus carpio), espécie que também pertence a família Cyprinidae como os kinguios. Segundo os autores precedentes, os juvenis de carpa tiveram o melhor ganho de peso nestes níveis de inclusão de vitamina $\mathrm{A}$, e redução da ingestão de alimento, perda de peso, presença de pontos hemorrágicos na pele e nadadeiras e empalidecimento da coloração, após 8 a 11 semanas de estudo, quando a exigência de vitamina A não foi atendida pela dieta fornecida. 
Tabela 3. Variáveis de desempenho produtivo de kinguios de acordo com os diferentes níveis de suplementação de vitamina A.

\begin{tabular}{|c|c|c|c|c|c|c|c|}
\hline \multirow{2}{*}{ Variáveis } & \multicolumn{6}{|c|}{ Tratamentos (U.I. de Vitamina A) } & \multirow{2}{*}{ C.V. $(\%)$} \\
\hline & Controle & 2.000 & 4.000 & 8.000 & 16.000 & 32.000 & \\
\hline Peso Inicial (g) & 6,66 & 6,65 & 6,67 & 6,67 & 6,66 & 6,68 & $1,51^{\mathrm{NS}}$ \\
\hline Ganho Peso Médioº (g) & $3,93^{\mathrm{bc}}$ & $4,99^{\mathrm{ab}}$ & $5,73^{\mathrm{a}}$ & $5,37^{\mathrm{ab}}$ & $4,55^{\mathrm{abc}}$ & $3,42^{\mathrm{c}}$ & $17,28^{*}$ \\
\hline Comprimento Padrão Médio ${ }^{2}(\mathrm{~cm})$ & $5,22^{\mathrm{b}}$ & $5,66^{\mathrm{a}}$ & $5,68^{\mathrm{a}}$ & $5,55^{\mathrm{ab}}$ & $5,66^{\mathrm{a}}$ & $5,37^{\mathrm{ab}}$ & $3,84^{*}$ \\
\hline Comprimento Total $\mathrm{Médio}^{3}(\mathrm{~cm})$ & $8,53^{\mathrm{ab}}$ & $9,01^{\mathrm{a}}$ & $8,85^{\mathrm{ab}}$ & $8,66^{\mathrm{ab}}$ & $8,72^{\mathrm{ab}}$ & $8,38^{\mathrm{b}}$ & $3,29^{*}$ \\
\hline Conversão Alimentar Aparente ${ }^{4}$ & $2,02^{\mathrm{c}}$ & $1,46^{\mathrm{ab}}$ & $1,36^{\mathrm{a}}$ & $1,21^{\mathrm{a}}$ & $1,19^{\mathrm{a}}$ & $1,86^{\mathrm{bc}}$ & $17,10^{*}$ \\
\hline Taxa de Cresc. Diário 5 (\%) & $0,51^{\mathrm{bc}}$ & $0,62^{\mathrm{abc}}$ & $0,68^{\mathrm{a}}$ & $0,65^{\mathrm{ab}}$ & $0,57^{\mathrm{abc}}$ & $0,46^{\mathrm{c}}$ & $14,10^{*}$ \\
\hline Sobrevivência (\%) & 95,83 & 97,67 & 100,00 & 97,67 & 100,00 & 100,00 & $7,44^{\mathrm{NS}}$ \\
\hline
\end{tabular}

*Médias na mesma linha seguidas de letras distintas diferem $(\mathrm{P}<0,05)$ pelo teste de Duncan.

${ }^{1}$ Efeito quadrático $\left(y=-0,0043 x^{2}+0,0970 x+4,6490 ; R^{2}=0,61\right)$;

${ }^{2}$ Efeito quadrático $\left(y=-0,0013 x^{2}+0,0364 x+5,4182 ; R^{2}=0,46\right)$

${ }^{3}$ Efeito quadrático $\left(y=-0,0007 x^{2}-0,0096 x+8,7423 ; R^{2}=0,73\right)$;

${ }^{4}$ Efeito quadrático $\left(y=0,0030 x^{2}-0,0928 x+1,7955 ; R^{2}=0,83\right)$;

${ }^{5}$ Efeito quadrátic0 $\left(y=-0,0004 x^{2}+0,0093 x+0,5841 ; R^{2}=0,63\right)$;

Fonte: Elaboração dos autores.

Figura 1. Ganho de peso médio de kinguios de acordo com os diferentes níveis de suplementação de vitamina A.

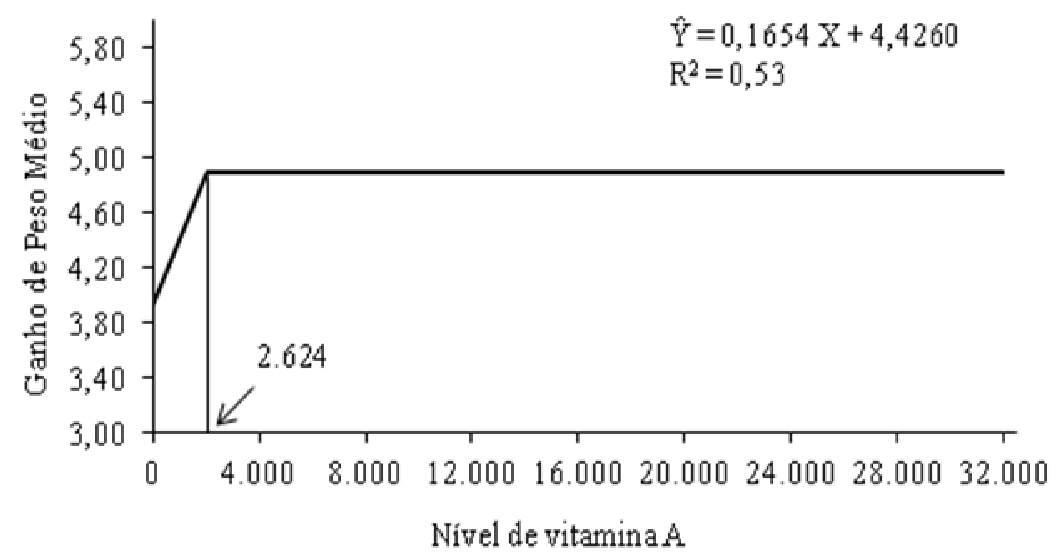

Fonte: Elaboração dos autores.

Através do modelo LRP observamos que o nível ideal de vitamina A para comprimento padrão e comprimento total médio está em 2.385 e 2.358 respectivamente, a partir do qual ocorre um platô (Figuras 2 e 3), correspondendo a um crescimento de 5,57 e 8,65 para comprimento padrão e comprimento total médio, respectivamente. O melhor nível de vitamina A em termos de conversão alimentar aparente também foi com 4.000 UI, embora não tenha apresentado diferenças significativas em relação aos níveis suplementação de 8.000 e 16.000 UI de vitamina A por $\mathrm{kg}$ de dieta. O modelo LRP estima para a conversão alimentar aparente um valor de ótimo em $4.381 \mathrm{UI}$ de vitamina A por $\mathrm{kg}$ de ração a partir do qual ocorre um platô (Figura 4), correspondendo a uma conversão alimentar aparente de 1,86 . 
Figura 2. Comprimento padrão médio de kinguios de acordo com os diferentes níveis de suplementação de vitamina A.

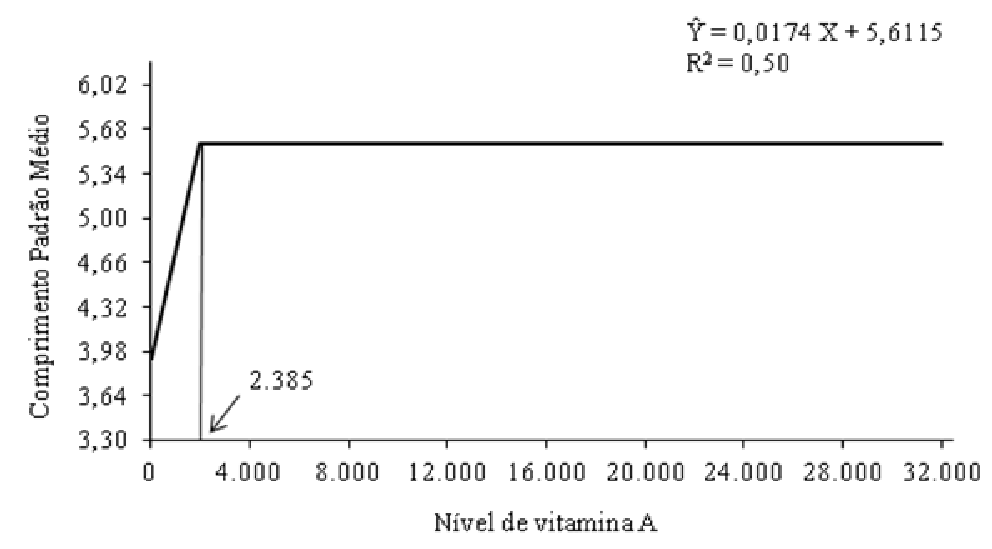

Fonte: Elaboração dos autores.

Figura 3. Comprimento total médio de kinguios de acordo com os diferentes níveis de suplementação de vitamina A.

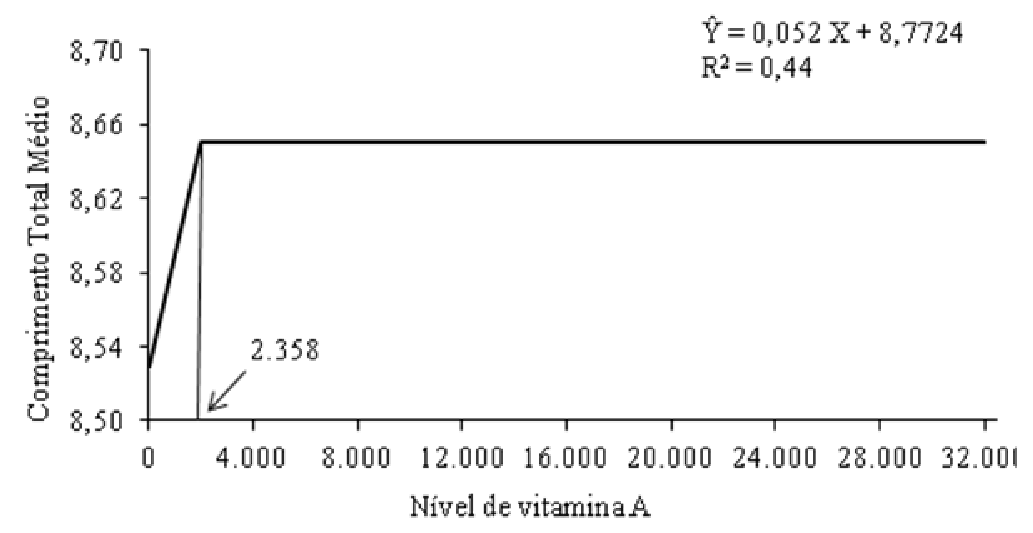

Fonte: Elaboração dos autores.

Figura 4. Conversão alimentar aparente de kinguios de acordo com os diferentes níveis de suplementação de vitamina A.

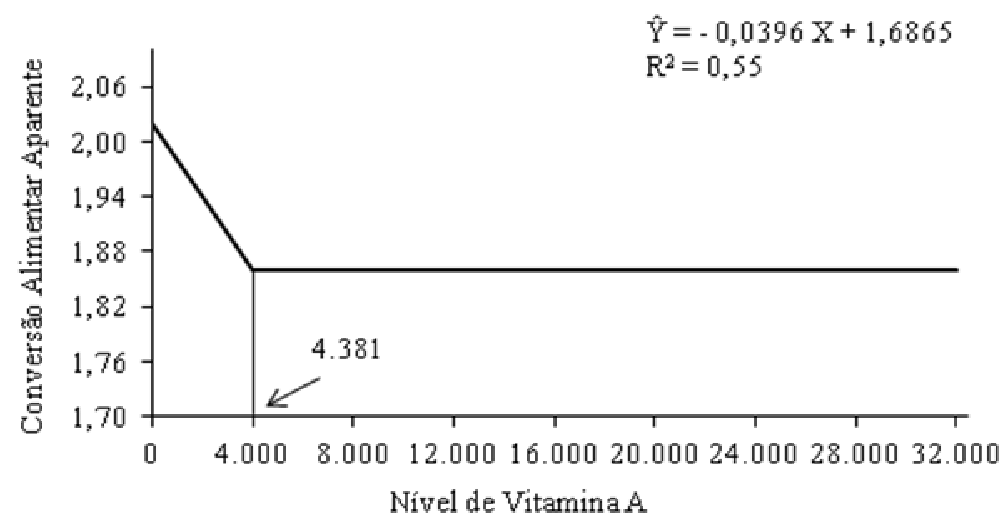

Fonte: Elaboração dos autores. 
Os peixes que receberam o maior nível de inclusão de vitamina A (32.000 UI) tiveram o pior desempenho em termos de crescimento e ganho de peso, condizente com alguns dos sinais de toxidade pelo excesso do nutriente na dieta. Segundo Tacon (1991), dentre os sintomas da hipervitaminose estão o crescimento reduzido, escoliose, lordose, fígado amarelo, necrose/erosão das nadadeiras e mortalidade. De maneira geral, estes sinais são parecidos com os apresentados em casos de deficiência, o que também pode ser percebido no presente estudo pela semelhança entre os dados obtidos dos animais que receberam a ração controle (sem suplementação de vitamina A) com aqueles que receberam $32.000 \mathrm{UI} \mathrm{kg}^{-1}$ de ração.

No modelo LRP para a taxa de crescimento diário a exigência é de 2.866 UI de vitamina A por $\mathrm{kg}$ de ração, nesse ponto ocorre um platô (Figura 5), correspondente a um crescimento diário de 0,52. Independente do nível de suplementação da vitamina A não houve efeito negativo sobre a sobrevivência dos animais, que foram estatisticamente semelhantes.

Figura 5. Taxa de crescimento diário de kinguios de acordo com os diferentes níveis de suplementação de vitamina A.

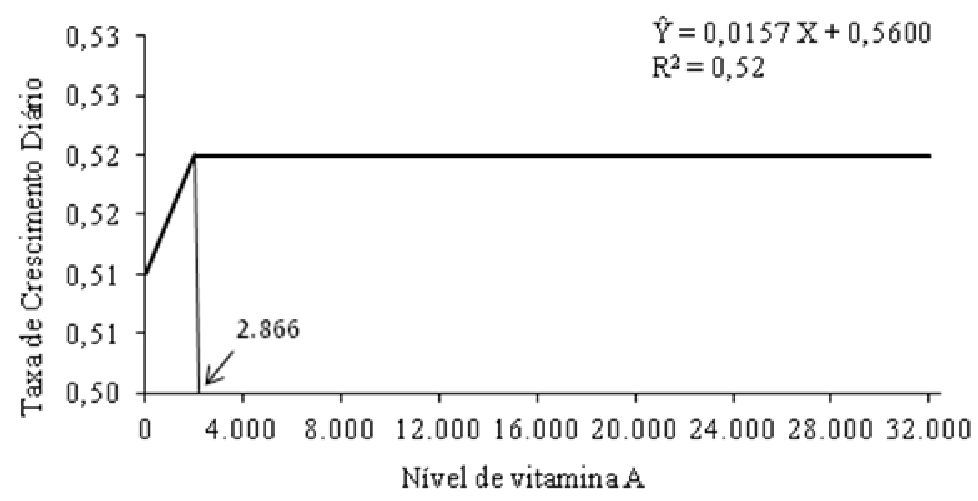

Fonte: Elaboração dos autores.

Não foram observados efeitos $(\mathrm{P}>0,05)$ da suplementação de vitamina A na dieta com reflexos na pigmentação da pele do kinguio (Tabela 4). Esta característica pode ter sido influenciada por fatores ambientais como a salinidade, intensidade de luz e temperatura da água. A falta de influência nesta característica pode estar associada à temperatura da água $\left(19,95 \pm 2,73{ }^{\circ} \mathrm{C}\right)$ e a alta transparência da água do sistema de cultivo, pois está permaneceu cristalina durante todo o período experimental.

Os peixes são animais ectotérmicos, logo, a temperatura do meio onde vivem altera o seu metabolismo, influenciando diretamente o consumo de alimento e o processo digestivo. Corroborando com Gouveia e Rema (2005), onde a fonte e a concentração de carotenoides influenciaram na pigmentação da pele de $C$. auratus, bem como o período de suplementação e composição da dieta.

Fatores como tamanho do peixe, idade, composição da dieta, período de suplementação, maturação sexual e fatores genéticos também influenciam a pigmentação da pele dos peixes (STOREBAKKEN; NO, 1992; GOUVEIA; REMA, 2005). A coloração dos peixes é controlada pelo sistema endócrino e nervoso, porém, fontes de pigmentos alimentares também podem exercer um papel na coloração dos peixes (MOYLE; $\mathrm{CECH}$, 1996). A glândula pituitária segrega hormônios que controlam a produção e armazenamento de pigmentos durante a vida do peixe, com acentuado 
aumento na maturidade sexual, podendo essa, ser a razão pela qual, várias espécies de peixes usam sua coloração para a camuflagem ou para atrair um parceiro (SINHA; ASIMI, 2007).

O múltiplo padrão de cores encontrado em peixes depende de interações entre os pigmentos nas células tegumentares, conhecido como cromatóforos (melanóforos, xantóforos, eritróforos, iridióforos, leucóforos e cianóforos), apresentando pigmentos como melanina, carotenóides (astaxantina, cantaxantina, luteína e zeaxantina), pteridinas e purinas (FUJII, 2000). Esse fato que propicia uma variedade de padrões de coloração como resultado da dispersão ou da agregação de cromatossomas e da distribuição dos cromatóforos na pele (FUJII, 2000). A alteração da intensidade da cor nos cromatóforos ocorre por meio da modificação na posição do pigmento no seu interior, tornando-o mais ou menos exposto para visualização (FUJII, 2000). A combinação de fatores ambientais, neurais, endócrinos e ao comportamento reprodutivo influencia na dispersão dos cromatossomas nos cromatóforos e na deposição dos pigmentos em peixes cultivados (ASPENGREN et al., 2003).

Tabela 4. Índices de coloração em Carassius auratus alimentados com dietas suplementadas com vitamina A por 88 dias.

\begin{tabular}{|c|c|c|c|c|c|c|c|}
\hline \multirow{2}{*}{$\begin{array}{l}\text { Índices de } \\
\text { Coloração }\end{array}$} & \multicolumn{6}{|c|}{ Nível de inclusão (U.I. de Vitamina A) } & \multirow{2}{*}{ C.V. $(\%)$} \\
\hline & Controle & 2.000 & 4.000 & 8.000 & 16.000 & 32.000 & \\
\hline $\mathrm{L}$ & 36,08 & 35,06 & 38,35 & 36,99 & 37,94 & 33,71 & 7,20 \\
\hline A & 22,52 & 25,36 & 24,61 & 18,51 & 22,86 & 23,43 & 15,18 \\
\hline $\mathrm{B}$ & 37,37 & 35,89 & 35,97 & 34,60 & 35,82 & 34,42 & 7,04 \\
\hline $\mathrm{H}_{\mathrm{ab}}$ & 58,97 & 54,84 & 55,62 & 62,03 & 57,36 & 55,84 & 6,66 \\
\hline $\mathrm{C}_{\mathrm{ab}}^{\mathrm{ab}}$ & 6,61 & 6,63 & 6,60 & 6,27 & 6,52 & 6,44 & 3,85 \\
\hline $\mathrm{C}$ & 0,64 & 0,62 & 0,62 & 0,65 & 0,64 & 0,64 & 3,83 \\
\hline M & 0,97 & 1,05 & 0,85 & 0,96 & 0,84 & 0,86 & 21,67 \\
\hline Y & 1,53 & 1,47 & 1,46 & 1,36 & 1,44 & 1,47 & 5,11 \\
\hline $\mathrm{K}$ & 0,61 & 0,60 & 0,57 & 0,61 & 0,58 & 0,65 & 6,90 \\
\hline
\end{tabular}

Fonte: Elaboração dos autores.

\section{Conclusão}

A adição de $2.624,2.385$ e $2.358,4.381$ e 2.866 UI de vitamina A por kg de ração, melhora o ganho de peso, comprimento padrão e total, conversão alimentar aparente e taxa de crescimento diário versus níveis de vitamina $\mathrm{A}$, respectivamente, sem, contudo exercer influência significativa na intensificação da coloração da pele de juvenis de kinguios. Inclusões de $32.000 \mathrm{UI} / \mathrm{kg}$ de dieta têm efeito negativo sobre o desempenho, estando provavelmente associado à hipervitaminose.

\section{Comitê de Ética}

Este estudo foi aprovado pelo Comitê de Ética na Experimentação Animal da Universidade Estadual do Oeste do Paraná (Unioeste), Campus de Cascavel, sob o protocolo número 5213, considerando seus aspectos éticos e metodológicos.

\section{Referências}

AOE, H. I.; MASUDA, T.; MIMURA, T.; SAITO, A. K. Requirement of young carp for vitamin A. Bulletin of the Japanese Society for the Science of Fish, Washington, v. 34, p. 959-964, 1968. 
ASPENGREN, S.; SKÖLD, H. N.; QUIROGA, G.; MARTENSSON, L.; WALLIN, M. Noradrenaline and Melatonin-mediated regulation of pigment aggregation in fish melanophores. Pigment Cell Research, Oxford, v. 16, n. 1, p. 59-64, 2003.

BITTENCOURT, F.; SOUZA, B. E.; BOSCOLO, W. R.; RORATO, R. R.; FEIDEN, A.; NEU, D. H. Benzocaína e eugenol como anestésicos para o quinguio (Carassius auratus). Arquivo Brasileiro de Medicina Veterinária e Zootecnia, Belo Horizonte, v. 64, n. 6, p. 1597-1602, 2012.

DUPREE, H. K. Dietary requirement of vitamin A acetate and beta carotene. Progress in sport fishery research. Washington, D.C: Resource Publication, 1970. p. 148150. (Bureau of Sport Fisheries and Wildlife, n. 88).

FUJII, R. The regulation of motile activity in fish chromatophores. Pigment Cell Research, Oxford, v. 13, n. 5 , p. 300-319, 2000.

GOUVEIA, L.; REMA, P. Effect of microalgal biomass concentration and temperature on ornamental goldfish (Carassius auratus) skin pigmentation. Aquaculture Nutrition, Oxford, v. 11, n. 1, p. 19-23, 2005.

HALVER, J. E. The vitamins. HALVER, J. E.; HARDY, R. W. (Ed.). Fish nutrition. 3. ed. USA: Elservier Science, 2002. p. 181-257.

HAYASHI, C.; BOSCOLO, W. R.; SOARES C. M.; BOSCOLO, V. R.; GALDIOLI, E. M. Uso de diferentes graus de moagem dos ingredientes em dietas para a tilápia do Nilo (Oreochromis niloticus L.) na fase de crescimento. Acta Scientiarum Animal Sciences, Maringá, v. 21, n. 3, p. 733-737, 1999.

HEMRE, G. I.; DENG, D. F.; WILSON, R. P.; BERNTSSEN, M. H. G. Vitamin A metabolism and early biological responses in juvenile sunshine bass (Morone chrysops x M. saxatilis) fed graded levels of vitamin A. Aquaculture, Amsterdam, v. 235, n. 1-4, p. 645-658, 2004.

HERNANDEZ, L. H. H.; TESHIMA, S. I.; ISHIKAWA, M.; ALAM, S.; KOSHIO, S.; TANAKA, Y. Dietary vitamin A requirements of juvenile Japanese flounder Paralichthys olivaceus. Aquaculture Nutrition, Oxford, v. 11, n. 1, p. 3-9, 2005.

HU, C. J.; CHEN, S. M.; PAN, C. H.; HUANG, C. H. Effects of dietary vitamin $A$ or $\beta$-carotene concentrations on growth of juvenile hybrid tilapia, Oreochromis niloticus x O. aureus. Aquaculture, Amsterdam, v. 253, n. 1, p. 602-607, 2006.

KITAMURA, S.; SUWA, T.; OHARA, S.; NAKAGAWA, $K$. Studies on vitamin requirements of rainbow trout. Requirement for vitamin A and deficiency symptoms.
Bulletin of the Japanese Society for the Science of Fish, Washington, v. 33, p. 1126-1131, 1967.

MCDOWELL, L. R. Vitamins in animal nutrition: comparative aspects to human nutrition. California: Academic Press Inc., 1989. 486 p.

MOREN, M.; OPSTAD, I.; BERNTSSEN, M. H. G.; ZAMBONINO INFANTE, J. L.; HAMRE, K. An optimum level of vitamin A supplements for Atlantic halibut (Hippoglossus hippoglossus L.) juveniles. Aquaculture, Amsterdam, v. 235, n. 1-4, p. 587-599, 2004.

MOYLE, P. B.; CECH, J. J. Behavior and communication. Davis: University of California, 1996. 196 p.

NATIONAL RESEARCH COUNCIL - NRC. Vitamins. Nutrient requirements of fish and shrimp. Washington: National Academy Press, 2011. p. 186-231.

NICKELL, D. C.; BROMAGE, N. R. The effect of dietary lipid level on variation of flesh pigmentation in rainbow trout. Aquaculture, Amsterdam, v. 161, n. 1-4, p. $237-251,1998$.

REZENDE, F. P.; VIDAL-JÚNIOR, M. V.; ANDRADE, D. R.; MENDONÇA, P. P.; SANTOS, M. V. B. Characterization of a new methodology based on the intensity of skin staining of ornamental fish with applications in nutrition. Journal of Agricultural Science and Technology, v. 2, p. 606-613, 2012.

SCHÜMER, U. La grande guida dell'aquario. Roma: Stampa Stige, 2002. 187 p.

SHAIKMOHAMED, J.; SIVARAM, V.; CHRISTOPHER ROY, T. S.; PETER MARIAN, M.; MURUGADASS, S.; RAFFIQ HUSSAIN, M. Dietary vitamin A requirement of juvenile greasy grouper (Epinephelus tauvina). Aquaculture, Amsterdam, v. 219, n. 1-4, p. 693-701, 2003.

SHIMENO, S. Yellowtail, Seriola quinqueradiata. In: WILSON, R. P. Handbook of nutrient requirements of finfish. Boca Raton: CRC Press, 1991. p. 181-191.

SILVA, A. S. T.; SCHULZ, U. H. Crescimento de Carassius auratus (Actinopterygii: Cypriniformes) em tanques com e sem abrigo. Acta Biológica Leopondensia, São Leopoldo, v. 28, n. 1, p. 42-45, 2006.

SINHA, A.; ASIMI, O. A. China rose (Hibiscus rosasinensis) petals: a potent natural carotenoid source for goldfish (Carassius auratus L.). Aquaculture Research, Oxford, v. 38, n. 11, p. 1123-1128, 2007.

STOREBAKKEN, T.; NO, H. Pigmentation of rainbow trout. Aquaculture, Amsterdam, v. 100, n. 1-3, p. 209229, 1992. 
TACON, A. G. J. Vitamin nutrition in shrimp and fish. In: AKIYAMA, D. M.; TAN, R. K. H. (Ed.). Proceedings of the aquaculture feed processing and nutrition workshop. Beijing: American Soybean Association, 1991. p. 100141.

UNIVERSIDADE FEDERAL DE VIÇOSA - UFV. SAEG - Sistema para análises estatísticas e genéticas. Versão 7.1. Viçosa, MG, 1997. 150 p. (Manual do usuário).
VIEIRA, S.; HOFFMANN, R. Estatística experimental. São Paulo: Atlas S.A., 1989. 179 p.

ZUANON, J. A. S.; SALARO, A. L.; FURUYA, W. M. Produção e nutrição de peixes ornamentais. Revista Brasileira de Zootecnia, Viçosa, MG, v. 40, p. 165-174, 2011. Suplemento Especial. 
